\title{
əImprovement of Solid Precipitation Measurements Using a Hotplate Precipitation Gauge
}

\author{
Julie M. Thériault, ${ }^{\mathrm{a}}$ Nicolas R. Leroux, ${ }^{\mathrm{a}}$ AND Roy M. RASMUSSEN ${ }^{\mathrm{b}}$ \\ ${ }^{a}$ Centre ESCER, Department of Earth and Atmospheric Sciences, Université du Québec à Montréal, Montreal, Quebec, Canada \\ ${ }^{\mathrm{b}}$ Research Applications Laboratory, NCAR, Boulder, Colorado
}

(Manuscript received 7 July 2020, in final form 10 November 2020)

\begin{abstract}
Accurate snowfall measurement is challenging because it depends on the precipitation gauge used, meteorological conditions, and the precipitation microphysics. Upstream of weighing gauges, the flow field is disturbed by the gauge and any shielding used usually creates an updraft, which deflects solid precipitation from falling in the gauge, resulting in significant undercatch. Wind shields are often used with weighing gauges to reduce this updraft, and transfer functions are required to adjust the snowfall measurements to consider gauge undercatch. Using these functions reduces the bias in precipitation measurement but not the root-mean-square error (RMSE). In this study, the accuracy of the Hotplate precipitation gauge was compared to standard unshielded and shielded weighing gauges collected during the WMO Solid Precipitation Intercomparison Experiment program. The analysis performed in this study shows that the Hotplate precipitation gauge bias after wind correction is near zero and similar to wind corrected weighing gauges. The RMSE of the Hotplate precipitation gauge measurements is lower than weighing gauges (with or without an Alter shield) for wind speeds up to $5 \mathrm{~m} \mathrm{~s}^{-1}$, the wind speed limit at which sufficient data were available. This study shows that the Hotplate precipitation gauge measurement has a low bias and RMSE due to its aerodynamic shape, making its performance mostly independent of the type of solid precipitation.
\end{abstract}

KEYWORDS: Atmosphere; Precipitation; Snowfall; Data processing; Instrumentation/sensors; Measurements

\section{Introduction}

Snowfall measurement is challenging due to the wide variety of snow hydrometeors and due to the large upstream disturbance of the airflow upstream of typical shield/gauge measurement systems commonly used (typically a catching/weighing gauge). Weighing gauges impact the accuracy of the measurement due to its alteration on the local flow field around the gauge and hence the trajectory of oncoming snowflakes that it is trying to measure (Nitu et al. 2018). Previous studies (e.g., Groisman et al. 1991; Yang et al. 1995; Thériault et al. 2012) have shown snowfall undercatch to increase with increasing wind speed as a result. In addition, observations show a significant variability in undercatch for a given wind speed due to the wide variety of snow crystal types present in the atmosphere (Yang et al. 1995), as well as with snowfall intensity (Colli et al. 2020). Accurately measuring snowfall precipitation is of importance for hydrological forecasting, water management, and avalanche prediction, and thus improving the accuracy of snowfall is a high priority.

Various instruments are deployed to measure snowfall (Rasmussen et al. 2012) and some are shown in Fig. 1. The OTT Pluvio and Geonor weighing gauges (Figs. 1a,b) are two of the most commonly used instruments. These gauges are equipped with sensors that measure the mass of precipitation and converts the signal into precipitation accumulation.

\footnotetext{
D Denotes content that is immediately available upon publication as open access.

Corresponding author: Julie M. Thériault, theriault.julie@ uqam.ca
}

Optical sensors can also measure the amount of precipitation but they require making many assumptions on the density and phase of the precipitation particle detected (Boudala et al. 2014). Finally, the mass of snowfall can be estimated from the latent heat released during melting of solid precipitation using a Hotplate precipitation gauge (Fig. 1c) (Rasmussen et al. 2011; Zelasko et al. 2018). The Hotplate precipitation gauge consists of two vertically stacked circular plates kept at a constant temperature. The measurement technique consists of using an electric current to maintain the temperature of an upper sensing plate and lower baseline plate the same and typically around $75^{\circ} \mathrm{C}$. If precipitation falls on the top plate, it melts and evaporates typically within a minute. The difference in power usage between the two plates is then proportional to the precipitation rate by using the latent heat of evaporation and melting to convert power usage to precipitation rate.

The collection efficiency (CE) is a factor commonly used to develop transfer functions to correct different gauge-shield configurations. It is defined as the ratio between the observed precipitation in a gauge/shield pair and the "truth" measurement. The truth is assumed to be measured by a weighing gauge installed in a Double Fence Intercomparison Reference (DFIR) with a single Alter (SA) in the middle of the inner fence (Fig. 1b) (e.g., Goodison et al. 1998). The DFIR is typically used instead of the WMO standard Bush gauge (Goodison et al. 1998) due to its transferability to various locations around the world. The DFIR tends to underestimate $5 \%$ of the precipitation with respect to the Bush gauge (Yang 2014).

In addition to the varying measurement techniques, meteorological factors, precipitation intensity, and the microphysics of precipitation can impact the amount of precipitation falling 


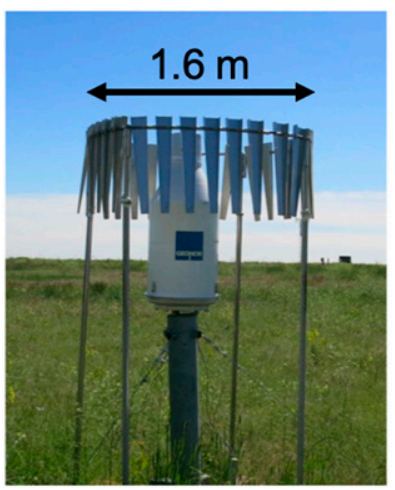

(a)

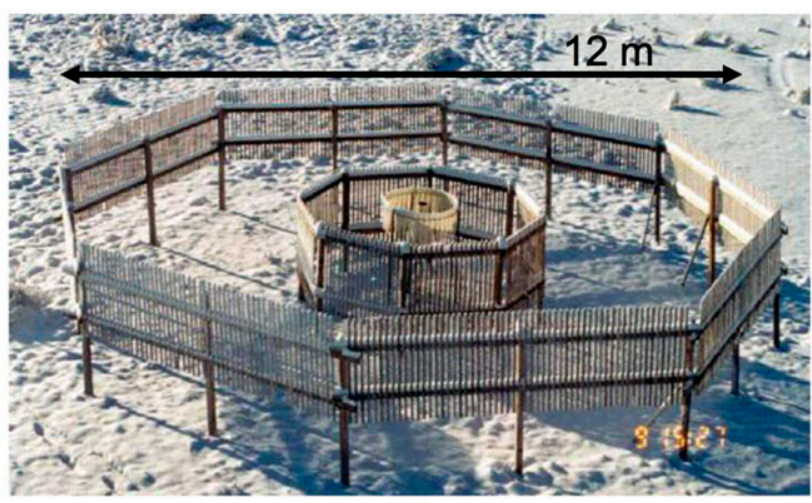

(b)

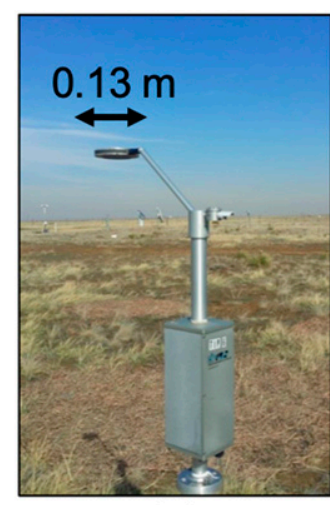

(c)

FIG. 1. (a) Geonor placed in a single-Alter (SA) shield, (b) a DFIR with a SA shielded Geonor in the middle, and (c) the Hotplate precipitation gauge (Rasmussen et al. 2011). Photos adapted from the WMO SPICE report (Nitu et al. 2018) and Rasmussen et al. (2011).

into the gauge (e.g., Rauch et al. 1998; Sevruk 1982, 1996; Thériault et al. 2012; Colli et al. 2020). The main problem associated with the measurement of solid precipitation by automatic gauges is the effect of the wind (Goodison et al. 1998). To reduce the wind effect on the collection efficiency of a gauge, different shields are installed around the weighing gauge with the goal of reducing the updraft created by the flow impacting the sidewalls of the gauge. But large differences in the amount of precipitation are still measured by the various gauge-shield configurations located at the same observational site (Rasmussen et al. 2012). The difference among the various methods to measure snowfall can be up to $110 \%$ (Yang et al. 2001).

This wind-induced error is fundamentally caused by the deformation of the flow in the vicinity of the gauge orifice, which creates an updraft that deflects particles approaching the gauge away from a straight-line trajectory, hence decreasing the collection efficiency of the gauge (e.g., Thériault et al. 2012; Rasmussen et al. 2012; Colli et al. 2016a,b). The shield around the gauge decreases the wind speed and, in turn, decreases the strength of the updraft upstream of the gauge orifice, resulting in an increase in collection efficiency. This has led to the popularity of the Alter shield, for example.

Transfer functions as a function of shield type and wind speed have been derived using data from various programs; most recently the WMO Solid Precipitation Intercomparison Experiment (WMO SPICE) program (Nitu et al. 2018). Despite the transfer function improving the collection efficiency for a given wind shield and wind speed, the scatter in the data at a given wind speed is still largely present even with correction with a transfer function. A scatter observed at wind speeds close to zero is likely due to technical factors, such as noise induced by the vibrating wires used by the Geonor to weigh the snow, as well spatial variation in snowfall rate relative to the truth DFIR system. As wind speed increases, the large scatter remains and has been explained by Thériault et al. (2012) and Leroux et al. (2021) by the variability of snow particle fall speed depending on snow particle type. It was shown by Thériault et al. (2012) using particle trajectory simulations that slower falling snowflakes $\left(\sim 1 \mathrm{~m} \mathrm{~s}^{-1}\right)$ tend to be deflected away from the gauge more than faster falling snowflakes $\left(>2 \mathrm{~m} \mathrm{~s}^{-1}\right)$.

To estimate the actual amount of precipitation accumulating on the ground, transfer functions have been developed to account for the impact of wind speed, air temperature, and precipitation intensity on the collection efficiency of weighing gauges (see, e.g., Yang et al. 1995; Colli et al. 2020). Wind speed is the main factor creating a disturbed flow upstream of the gauge, and thereby impacting the snow particle trajectory. Air temperature is used as a proxy to estimate the phase of the precipitation (e.g., Yang et al. 1995; Wolff et al. 2015). During the WMO SPICE program in 2013-15 (Nitu et al. 2018), transfer functions were developed and tested by Wolff et al. (2015) and Kochendorfer et al. (2017a,b, 2018) at the WMO SPICE sites. They found that correcting precipitation measurements with the newly developed transfer functions, using wind and temperature, produced a bias close to $0 \mathrm{~mm}$ but the RMSE remained relatively high and similar to that estimated prior to applying the transfer function. This relatively high RMSE after adjusting the measured precipitation adds significant uncertainty to the estimation of snowfall rate even when using a transfer function. In the following, we show that a Hotplate precipitation gauge can reduce a significant amount of the uncertainty in the RMSE while maintaining near zero bias due to its aerodynamic shape reducing the vertical velocity created by the ambient wind impacting the thin sidewalls of the gauge.

Given the importance of accurately measuring snowfall, it is critical to better understand the uncertainty associated with the standard precipitation gauge (shielded and unshielded) as well as the ones based on the relatively new technology of the Hotplate precipitation gauge. The goal of this study is to compare the accuracy of the standard weighing precipitation gauges (both unshielded and shielded) to the performance of the Hotplate precipitation gauge using WMO SPICE data (Nitu et al. 2018) collected at the Marshall Site, Boulder, Colorado, where Hotplate precipitation gauge and other gauge-shield configurations are available. A statistical analysis 
TABLE 1. Coefficients of the transfer functions [Eqs. (1) and (2)] and of the linear regression used to correct the precipitation measurements from the Hotplate gauge; $U$ is the $10-\mathrm{m}$ wind speed and $T$ is the $2-\mathrm{m}$ air temperature. In the linear regression, $x$ is the unadjusted HP measurements and $y$ the adjusted measurements.

\begin{tabular}{cccc}
\hline \hline & $f(U, T)$ [Eq. (1)] & $f(U)$ [Eq. (2)] & Linear regression $y=a x+b$ \\
\hline$a$ & -0.034 & 0.28 & 0.78 \\
$b$ & -0.16 & 0.41 & $0.078 \mathrm{~mm}$ \\
$c$ & 0.15 & 1.0 & - \\
\hline
\end{tabular}

of the relative amount of precipitation measured by these gauges is conducted.

The paper is organized as follows: the methodology is described in section 2, a comparison of the different gauge-shield configurations is given in section 3, a statistical analysis is presented in section 4, and discussion and conclusions are given in section 5 .

\section{Methodology}

The WMO SPICE dataset presented in Kochendorfer et al. (2017a,b) was analyzed in this study but only solid precipitation events (air temperature $T<-2^{\circ} \mathrm{C}$ ) were considered. This study focuses on the Marshall Test site, Boulder, Colorado, as this site represents a typical midcontinental, midlatitude site. Details about the Marshall site can be found in Rasmussen et al. (2011) and Nitu et al. (2018).

\section{a. WMO SPICE datasets}

The WMO SPICE dataset (Kochendorfer et al. 2017a,b, 2018) contains 30-min precipitation events over the $2013 / 14$ and 2014/15 cold seasons that have been created based on extensive quality control and event selection (Kochendorfer et al. 2017a,b). This dataset includes 30-min precipitation amounts measured by a Geonor in the DFIR (hereafter referred to as DFIR), the Geonor in the single Alter (hereafter referred to as $\mathrm{SA}$ ), the unshielded Geonor (hereafter referred to as UN), and a Hotplate precipitation gauge (hereafter referred to as HP), as well as the 2-m air temperature, and 10-m wind speed and wind direction. The wind speed at gauge height $(\sim 2 \mathrm{~m})$ was estimated following Kochendorfer et al. (2017a,b), i.e., using a log-law profile with a roughness length of $1 \mathrm{~cm}$ and displacement height of $40 \mathrm{~cm}$.

The algorithm to estimate the precipitation rate from the raw output of the HP gauge is described in Rasmussen et al. (2011). This algorithm includes calibrated parameters that were determined using field measurements. The precipitation rates were initially corrected for wind speed following a linear correction equation presented in Rasmussen et al. (2011). The wind speed used for the linear correction was measured by the Hotplate precipitation gauge itself. This wind speed correction is done to account for snow particle sliding off the plate during high wind speeds. Zelasko et al. (2018) pointed out some uncertainties in the calibrated coefficients used in the algorithm of Rasmussen et al. (2011) to obtain the precipitation rates from raw outputs. Therefore, in this study, we denote the HP
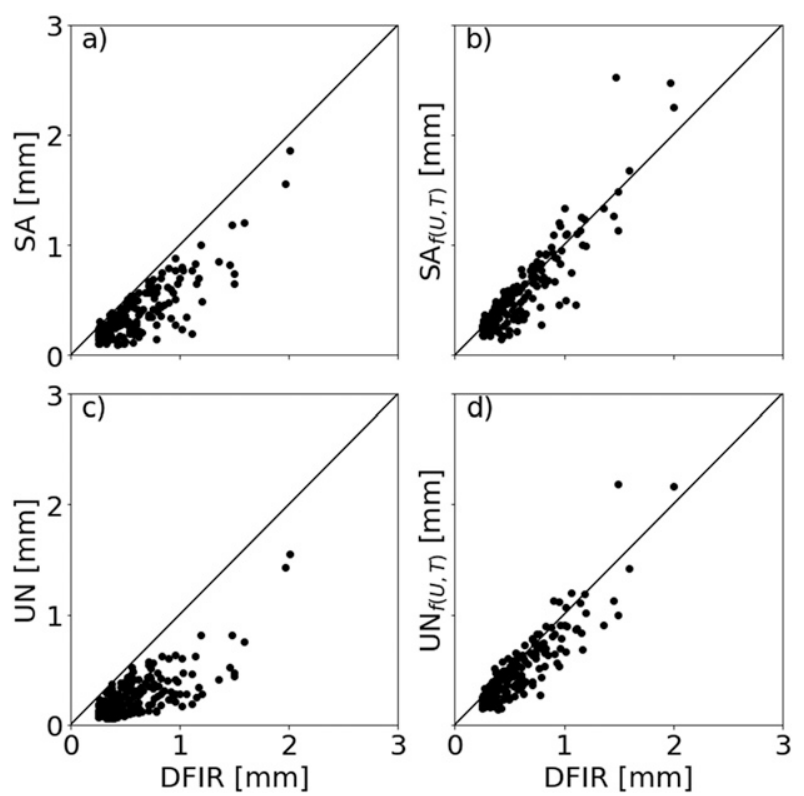

FIG. 2. Comparison of the 30-min accumulated precipitation with (a) the DFIR and the SA, (b) the DFIR and the SA corrected using the 10-m wind speed and 2-m air temperature $\left(\mathrm{SA}_{f(U, T)}\right)$, (c) the DFIR and the UN, and (d) the DFIR and the UN corrected using the $10-\mathrm{m}$ wind speed and $2-\mathrm{m}$ air temperature $\left(\mathrm{UN}_{f(U, T)}\right)$. Only solid precipitation $\left(T<-2^{\circ} \mathrm{C}\right)$ is considered. SA and UN denote the Geonor in the single-Alter shield and the unshielded Geonor, respectively. This figure differs from the one in Kochendorfer et al. (2017b), who used the same data but for all precipitation types.

precipitation rates estimated from the algorithm and the corrections of Rasmussen et al. (2011) as the unadjusted data, which are used as baseline for our analysis. Then, these unadjusted data will be further corrected for wind speed.

\section{b. Wind and precipitation data analysis}

The WMO SPICE data were filtered based on meteorological factors and subject to a minimum amount of precipitation accumulated of $0.25 \mathrm{~mm}$ by the DFIR over the $30-\mathrm{min}$ period. We applied the same filtering method proposed by WMO SPICE described in Kochendorfer et al. (2017a,b); the reader is referred to the original publications for additional details. Following this methodology but only for solid precipitation (air temperature $<-2^{\circ} \mathrm{C}$ ), a total of 211, 210, and 192 thirty-minute events were available to analyze measurements from the SA, UN, and HP, respectively. The collection efficiencies $(\mathrm{CE}$, the ratio of measured precipitation by the gauge under test and the DFIR) of the $\mathrm{UN}$ and the SA were determined using the $10-\mathrm{m}$ wind speed and the 2-m air temperature with Eq. (1). The transfer function used for the SPICE data by Kochendorfer et al. (2017b) is

$$
\mathrm{CE}=\exp \left(-a U\left\{1-\left[\tan ^{-1}(b T)+c\right]\right\}\right),
$$

where $a, b$, and $c$ are given in Kochendorfer et al. (2017b) (Table 1$), U$ is the $10-\mathrm{m}$ wind speed $\left(\mathrm{m} \mathrm{s}^{-1}\right)$, and $T$ is the $2-\mathrm{m}$ air temperature $\left({ }^{\circ} \mathrm{C}\right)$. 

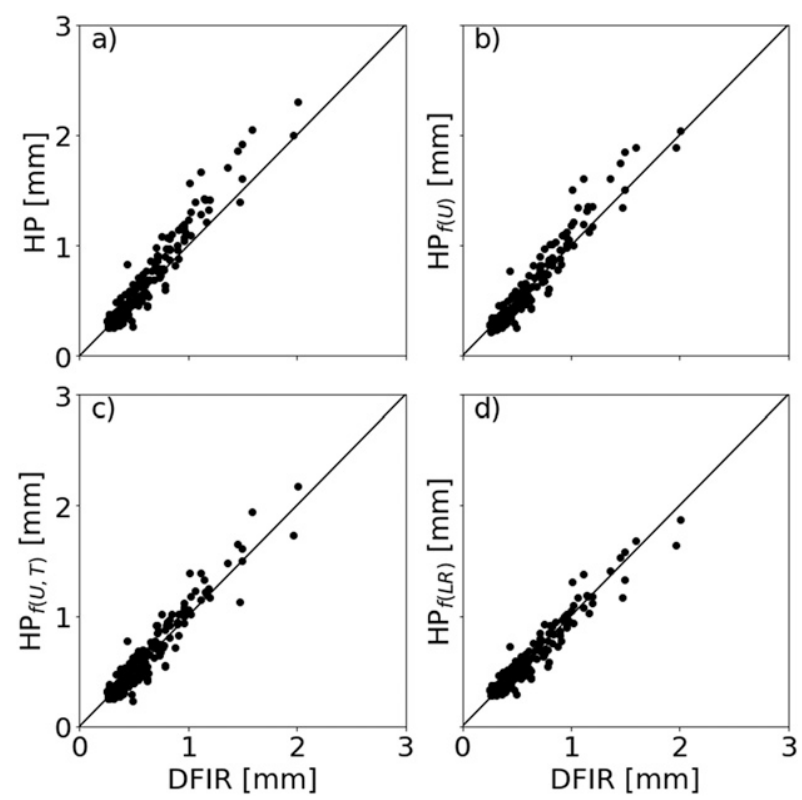

FIG. 3. Comparison of the 30-min accumulated precipitation with the DFIR and (a) the HP, (b) the adjusted HP precipitation amount with respect to the wind speed $\left(\mathrm{HP}_{f(U)}\right)$, (c) the adjusted HP precipitation amount with respect to wind speed and temperature $\left(\mathrm{HP}_{f(U, T)}\right)$, and (d) the adjusted HP precipitation using a linear regression $\left(\mathrm{HP}_{f(L R)}\right)$. Only solid precipitation is considered $\left(T<-2^{\circ} \mathrm{C}\right)$. HP denotes the Hotplate precipitation gauge.

Transfer functions for the HP were developed in the current study in three different ways: 1) using the 10-m wind speed and the 2-m air temperature [Eq. (1)], 2) solely using the 10-m wind speed [Eq. (2)], and 3) using a linear regression between the unadjusted HP and DFIR measurements (see Table 1 for the fitted coefficients). The transfer function using wind speed alone uses the following equation (Kochendorfer et al. 2018):

$$
\mathrm{CE}=a e^{-b U}+c,
$$

where the values of the coefficients $a, b$, and $c$ derived in our analysis are given in Table 1.

To estimate the parameters of the first two transfer functions [Eqs. (1) and (2)] for the HP, a tenfold cross-validation was used, i.e., the transfer functions were fitted independently in 10 iterations, using $90 \%$ of the data to train the model and $10 \%$ to test the model. The fitting coefficients of the two first transfer functions for the HP (Table 1) were taken as the average of the coefficients found during each of the tenfold cross validations. A threshold of $9 \mathrm{~m} \mathrm{~s}^{-1}$ was applied for the $10-\mathrm{m}$ wind speeds used in the transfer functions, i.e., wind speeds above this threshold were kept equal to the threshold (Kochendorfer et al. 2017a).

Measured and estimated precipitation from the SA, the UN, and the HP were compared to the DFIR measurements using the root-mean-square error (RMSE), bias and the correlation coefficient $\left(R^{2}\right)$. For the HP, the RMSE, bias, and $R^{2}$ were taken as the average of the values found in each of the 10 iterations for the cross validation.

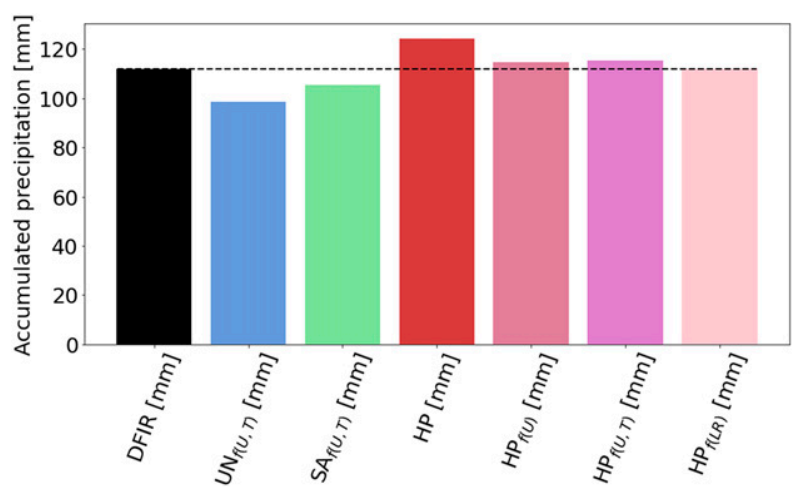

FIG. 4. Comparison of the total amount of precipitation measured by the different instruments (raw and adjusted) for $T<-2^{\circ} \mathrm{C}$ for the common 30-min events to all the instruments. The dashed line shows the accumulated precipitation measured by the Geonor in the DFIR. SA, UN, and HP denote the Geonor in the single Alter, the unshielded Geonor, and the Hotplate precipitation gauge, respectively.

\section{Comparison of the measured amount of precipitation}

The 30-min precipitation amounts measured by the UN and SA (unadjusted and adjusted) are compared with the DFIR in Fig. 2. The results are slightly different from those of Kochendorfer et al. (2017b) because only solid precipitation is studied here. The adjusted precipitations shown in Figs. $2 \mathrm{~b}$ and $2 \mathrm{~d}$ are based on Eq. (1), which considers both the 2-m air temperature and the $10-\mathrm{m}$ wind speed. Unadjusted precipitation amounts are underestimated by both the UN and the SA with respect to the DFIR (Figs. 2a,c), whereas the adjusted precipitations are closer to the DFIR measurements (Figs. 2b,d). Despite the improvements made by correcting the measurements with the transfer function [Eq. (1)], the adjusted SA and UN measurements are mainly underestimated for 30-min DFIR measurements $<1.5 \mathrm{~mm}$, whereas they were greatly overestimated when the DIFR measured more than $1.5 \mathrm{~mm}$ over $30 \mathrm{~min}$.

The adjusted and unadjusted amounts of precipitation measured by the HP are compared with the amount of precipitation measured by the DFIR in Fig. 3. For the unadjusted data (Fig. 3a), the scatter in the data is smaller than for SA and UN (Fig. 2). The HP gauge with the correction of Rasmussen et al. (2011) generally overestimates the 30-min true precipitation, particularly for higher amounts of precipitation $(>1.2 \mathrm{~mm})$. Figures 3b-d compare the adjusted precipitation amounts for the HP with the precipitation measured in the DFIR using Eq. (1) $[f(U)]$, Eq. (2) $[f(U, T)]$, and a linear regression $[f(\mathrm{LR})]$, respectively. In comparison to the adjusted measurements from SA and UN (Figs. 2b and 2d, respectively), the adjusted precipitation from HP (Figs. 3b-d) are more closely centered around the 1:1 line.

Over the 30-min events common to all the instruments, the total amount of precipitation measured by both the UN and SA gauges are 50.2 and $72.8 \mathrm{~mm}$, respectively, and the adjusted amounts increased to $86.0 \mathrm{~mm}$ (increase of $171 \%$ ) and $94.8 \mathrm{~mm}$ (increase of $130 \%$ ) (Fig. 4); as a reference, the DFIR 


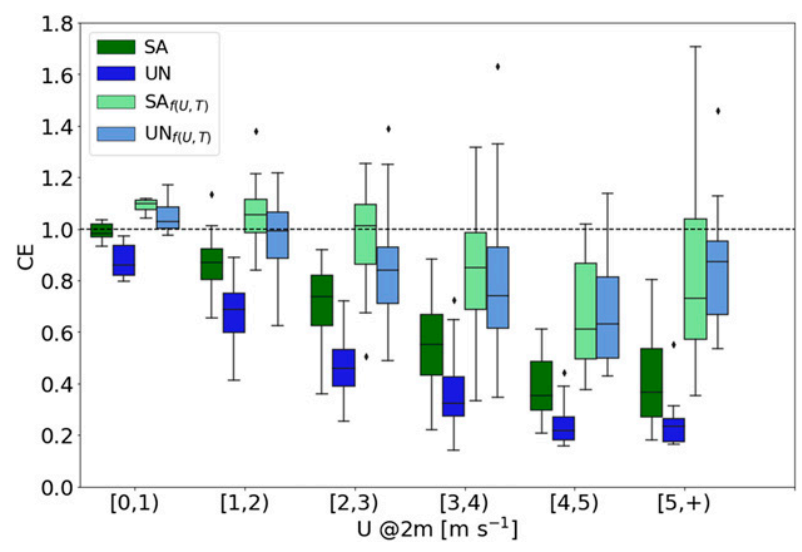

FIG. 5. CE of the UN and SA with respect to the DFIR using 30-min sample as well as solid precipitation $\left(T<-2^{\circ} \mathrm{C}\right)$ for different wind speeds $U$ at the gauge height. The adjusted UN $\left(\mathrm{UN}_{f(U, T)}\right)$ and SA $\left(\mathrm{SA}_{f(U, T)}\right)$ are computed using (2) using the $10-\mathrm{m}$ wind speed and 2-m air temperature. SA and UN denote the Geonor in the single-Alter shield and the unshielded Geonor, respectively. Here, $[0,1)$ means $\geq 0$ and $<1 \mathrm{~m} \mathrm{~s}^{-1}$.

measured a total amount of precipitation of $110.4 \mathrm{~mm}$. The total amount of precipitation measured by UN is less than the amount measured by SA due to the positive impact of the shield on measuring precipitation. Furthermore, the Hotplate precipitation gauge measures a total of $121.7 \mathrm{~mm}$. For the adjusted HP precipitation measurements with respect to wind speed only, both wind speed and temperatures, using the linear regression, the total amount of precipitation is $113.0,113.9$, and $110.0 \mathrm{~mm}$, respectively. The corrected HP measurements differed by less than $10 \%$ from the unadjusted HP precipitations.

\section{Comparison of collection efficiency}

The variation of $\mathrm{CE}$ with wind speed is a useful variable to compare between gauges to assess the performance and the limitations of each instrument and of the adjustments performed to the data.

$\mathrm{CE}$ of the adjusted and unadjusted measurements from the WMO SPICE unshielded and shielded gauges at the Marshall site are compared in Fig. 5. The number of events in each wind speed bin is given in Table 2. CE of the UN decreases rapidly with wind speed and reaches a plateau at 0.2. A similar pattern is observed for the Geonor placed in an SA, but the CE of SA is higher than the CE of UN. The adjusted precipitation amounts produced a higher 50th percentile (closer to 1 ) but the scatter of $\mathrm{CE}$ at a given wind speed bin increases. This is the case for both UN and SA. Note that for wind speeds larger than $5 \mathrm{~m} \mathrm{~s}^{-1}$, the scatter in the adjusted CE produces collection efficiencies above 1.4 in some cases.

CE of the unadjusted HP is compared to adjusted CE of UN and SA in Fig. 6. CE of the unadjusted HP at a given wind speed is always higher than the adjusted precipitation of UN and SA. The scatter (CE between the whiskers) is much higher between 0 and $1 \mathrm{~m} \mathrm{~s}^{-1}$ for the HP but remains approximately
TABLE 2. Number of 30-min samples for each 2-m wind speed bin for each instrument. $[0,1)$ means $\geq 0$ and $<1 \mathrm{~m} \mathrm{~s}^{-1}$. SA is the Geonor within the single-Alter shield, UN is the unshielded Geonor, and HP is the Hotplate precipitation gauge.

\begin{tabular}{crrr}
\hline \hline 2-m wind speed bin $\left(\mathrm{m} \mathrm{s}^{-1}\right)$ & SA & UN & HP \\
\hline$[0,1)$ & 6 & 6 & 4 \\
{$[1,2)$} & 46 & 46 & 44 \\
{$[2,3)$} & 73 & 73 & 66 \\
{$[3,4)$} & 53 & 51 & 48 \\
{$[4,5)$} & 19 & 21 & 18 \\
{$[5,+)$} & 13 & 14 & 12 \\
\hline
\end{tabular}

constant with increasing wind speed. This large amount of scatter at low wind speeds is consistent with Hotplate results from the Sodankyla site in Finland for SPICE where a Hotplate was deployed under low wind speed conditions (Nitu et al. 2018). This scatter was attributed to the heat plume generated by the top plate of the Hotplate interfering with the straightline snow particle trajectories. For all wind speed bins, the unadjusted HP slightly overestimates the amount precipitation measured but the standard deviation for a given wind speed is significantly smaller than that of the UN and SA. CE of the unadjusted HP initially decreases with increases wind speed and then increases for wind speeds $\geq 5 \mathrm{~m} \mathrm{~s}^{-1}$. This behavior is consistent with the numerical simulations of Cauteruccio (2020) and is caused by both the geometry of the HP and the flow disturbance induced by the gauge geometry. The top rings present on the edge of the HP can prevent some particles from falling onto the plate while the inner top rings result in additional particles being captured by the HP. This undercatch and overcatch of particles vary with wind speed, as shown in Cauteruccio (2020).

The CE of the adjusted precipitation from the Hotplate using the three different methods discussed in section $2 b$ is shown in Fig. 7. For the adjusted Hotplate measurements, CE is close to 1 ; for wind speeds $<3 \mathrm{~m} \mathrm{~s}^{-1}$, the adjusted HP measurements are better using the $10-\mathrm{m}$ wind speed, and for higher wind speeds, the adjusted precipitations using the linear regression is closer to the DFIR.

\section{Variability of precipitation estimation using various gauge types after applying a transfer function}

The variability of the precipitation estimates by the different gauges is represented by the RMSE as a function of wind speed (Fig. 8a). Table 2 presents the number of samples for each instrument and each wind speed bin. For $2-\mathrm{m}$ wind speeds $<1 \mathrm{~m} \mathrm{~s}^{-1}$, the RMSE is less than $0.1 \mathrm{~mm}$ for all precipitation estimates and this value increases with increasing wind speed; this increase agrees with the increase of the scatter in precipitation amounts with wind speed from the unadjusted/adjusted gauges and the DFIR (Figs. 2 and 3). This increase in RMSE with wind speed is less for the HP, in particular for the adjusted amounts. For the HP, the RMSE remains below $0.1 \mathrm{~mm}$ for wind speeds up to $5 \mathrm{~m} \mathrm{~s}^{-1}$, then it increases to about $0.4 \mathrm{~mm}$ at wind speeds $\geq 5 \mathrm{~m} \mathrm{~s}^{-1}$. These values are up to 4 times lower at $4 \mathrm{~m} \mathrm{~s}^{-1}$ and 2 times lower at wind speeds $>$ $6 \mathrm{~m} \mathrm{~s}^{-1}$ than for the unadjusted precipitation measurements 


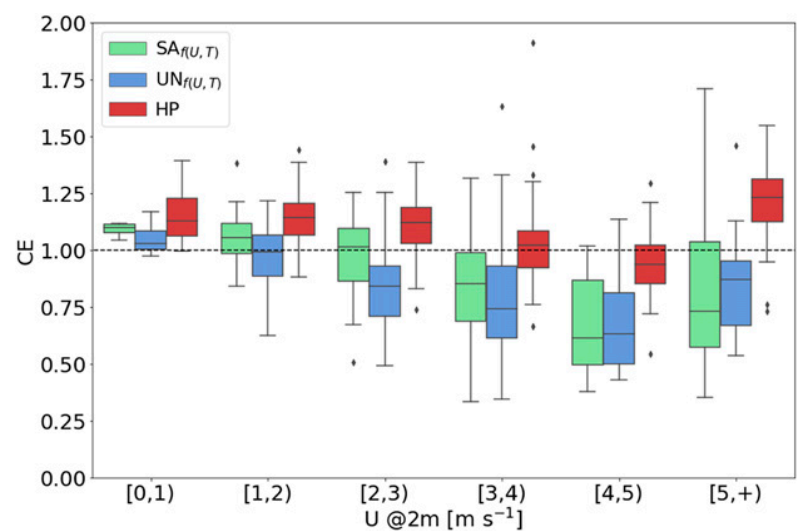

FIG. 6. CE of the adjusted UN $\left(\mathrm{UN}_{f(U, T)}\right)$, adjusted SA $\left(\mathrm{SA}_{f(U, T)}\right)$, and unadjusted $\mathrm{HP}$ using 30-min sample as well as solid precipitation $\left(T<-2^{\circ} \mathrm{C}\right)$ for different wind speeds $\mathrm{U}$ at the gauge height. The transfer function for precipitation correction [Eq. (2)] used the 10-m wind speed and the 2-m air temperature. SA, UN, and HP denote the Geonor in the single-Alter shield and the unshielded Geonor, and the Hotplate precipitation gauge, respectively. Here, $[0,1)$ means $\geq 0$ and $<1 \mathrm{~m} \mathrm{~s}^{-1}$.

with the weighing gauges. Adjusting the precipitation amounts with the transfer functions presented in section $2 \mathrm{~b}$ results in a decrease in the RMSE for all the instruments. The adjusted HP measurements generally have a lower RMSE than the adjusted UN and SA. In particular, at wind speeds $\geq 2 \mathrm{~m} \mathrm{~s}^{-1}$, the RMSE of the unadjusted HP was lower than the adjusted UN and SA Geonors.

The precipitation biases also vary with wind speed (Fig. 8b). The weighing precipitation gauges have a low bias for wind speeds $<1 \mathrm{~m} \mathrm{~s}^{-1}$. The bias decreases (increases in absolute value) for the SA and UN gauges as wind speed increases. For the HP gauge, the bias is systematically lower than for the UN and SA gauges. The absolute bias for the precipitation measured by the HP is less than $<0.1 \mathrm{~mm}$ for wind speeds $<5 \mathrm{~m} \mathrm{~s}^{-1}$. At wind speed $>5 \mathrm{~m} \mathrm{~s}^{-1}$, the bias of the Hotplate increases to almost $0.25 \mathrm{~mm}$ but the bias associated with the adjusted HP using the linear regression correction is near $0 \mathrm{~mm}$. Overall, even if the bias is improved for the UN and SA gauges for increasing wind speed using the transfer function [Eq. (1)], the bias associated with precipitation measurements by the HP is lower using the algorithm of Rasmussen et al. (2011) and near $0 \mathrm{~mm}$ when adjusted using a linear regression.

\section{Summary and conclusions}

\section{a. Summary}

The accuracy of standard precipitation gauges was investigated using the WMO Solid Precipitation Intercomparison Experiment (SPICE) datasets collected at the Marshall Test site in Boulder, Colorado. This test site was chosen because a Hotplate precipitation gauge was installed at the site during the experiment and included in the official WMO SPICE dataset. We used 211, 210, and 192 samples for the unshielded Geonor

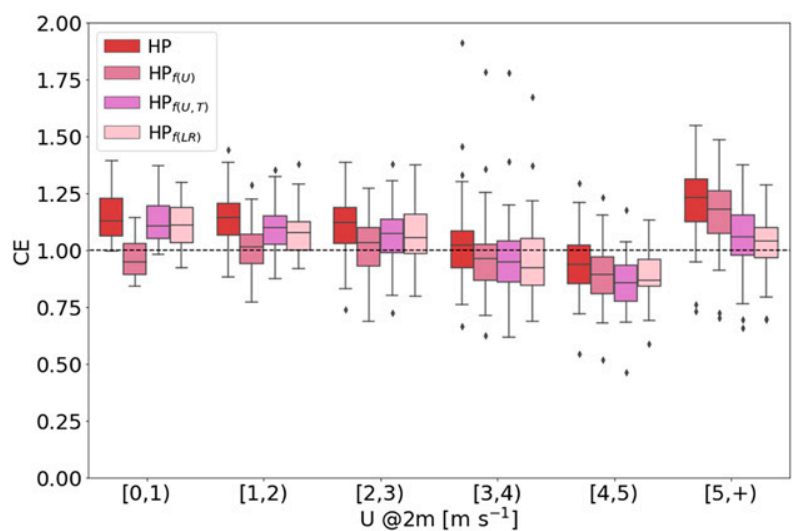

FIG. 7. CE of the raw and adjusted HP using 30-min sample for solid precipitation $\left(T<-2^{\circ} \mathrm{C}\right)$ for different wind speeds $U$ at the gauge height. The unadjusted HP measurements (red whisker boxes, HP) followed the wind correction of Rasmussen et al. (2011), who used the internal wind measurements of the HP precipitation gauge. The transfer functions used for wind speed adjustment is Eq. (3) $\left(\mathrm{HP}_{f(U)}\right)$ and for wind speed and temperature adjustment is Eq. (2) $\left(\mathrm{HP}_{f(U, T)}\right)$. The HP measurements were also adjusted following a linear regression $\left(\mathrm{HP}_{f(\mathrm{LR})}\right)$ between the unadjusted HP measurements and the precipitation measurement by the DFIR. HP denotes the Hotplate precipitation gauge. Here, [0, 1) means $\geq 0$ and $<1 \mathrm{~m} \mathrm{~s}^{-1}$.

gauge, shielded Geonor gauge, and Hotplate precipitation gauge, respectively. While the transfer function developed in previous studies to correct for wind undercatch for the unshielded and shielded Geonor gauges improved the precipitation bias, RMSE remains relatively high for the unshielded and shielded Geonors after wind correction (Fig. 9). The RMSE and bias of the unadjusted Hotplate precipitation measurements, however, are significantly less than those of both the adjusted shielded and unshielded gauges. The bias is near $0 \mathrm{~mm}$ for the adjusted Hotplate precipitation measurements (Fig. 9).

The Hotplate measurements corrected by a simple linear regression equation produced slightly lower RMSE and bias than for the adjusted Hotplate measurements with the transfer function using wind speed [Eq. (3)] and both wind speed and temperature [Eq. (2)] (Fig. 9). This is likely because the size distribution and fall speed of particles do not impact the collection efficiency of the Hotplate precipitation gauge as much as for the unshielded and shielded Geonors (Cauteruccio 2020) due to the aerodynamic profile presented by the Hotplate precipitation gauge to the oncoming flow. Cauteruccio (2020) estimated an overcatch of the snowfall precipitation measured by the HP for reference wind speeds $>5 \mathrm{~m} \mathrm{~s}^{-1}$ and used polynomial regressions for adjusting the Hotplate precipitation gauge data.

The amount of precipitation measured by the different gauges during no wind conditions is a good indicator of the performance of the gauges. During no wind conditions, any type of precipitation representing a variety of fall speeds should fall in the gauge because there is no deflection of the wind upstream and thus the uncertainty associated with the gauge can be assessed using those conditions. This is 

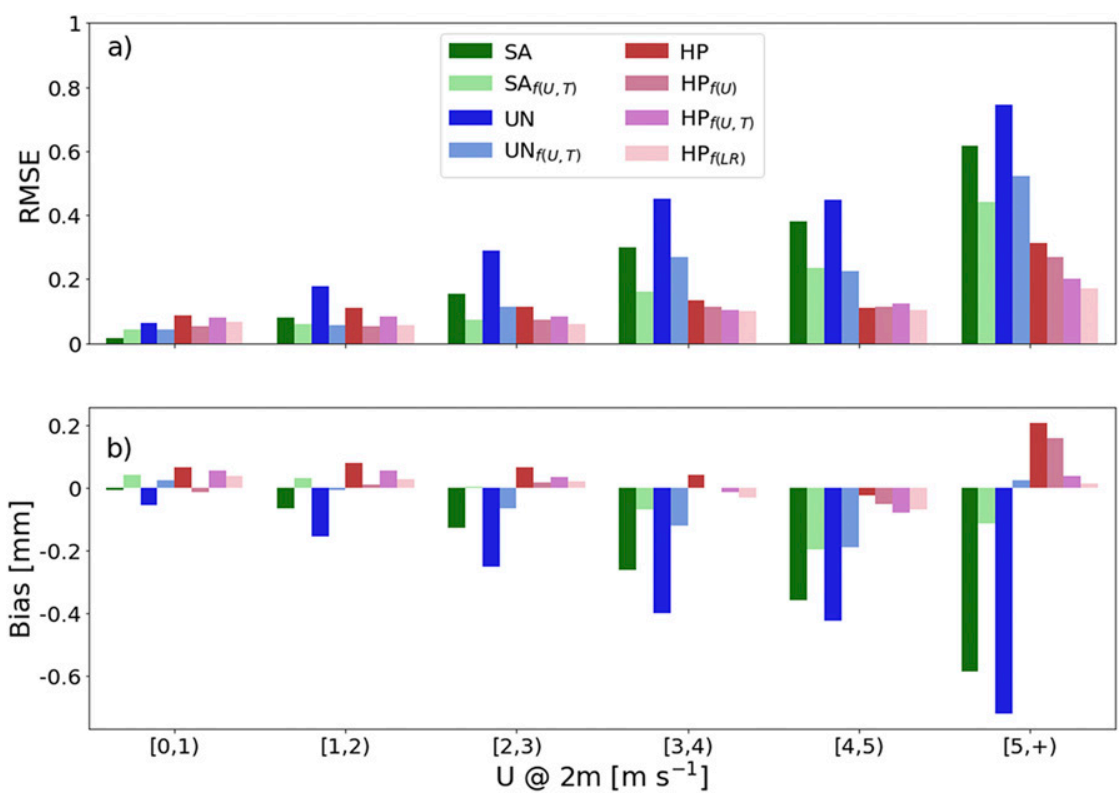

FIG. 8. (a) RMSE between the amount of precipitation measured by each instrument (unadjusted and adjusted) and the DFIR $\left(T<2^{\circ} \mathrm{C}\right)$ and (b) bias between the amount of precipitation measured by each instrument (unadjusted and adjusted) and the DFIR $\left(T<2^{\circ} \mathrm{C}\right)$ varying with wind speed $U$ at gauge height. $\mathrm{UN}$ and $\mathrm{UN}_{f(U, T)}$ denote the unadjusted and adjusted measurements of the unshielded Geonor, respectively. The same notation is used for the Geonor in the single-Alter shield (SA) and the Hotplate precipitation gauge (HP). The unadjusted HP measurements (red bars, HP) followed the wind correction of Rasmussen et al. (2011), who used the internal wind measurements of the HP precipitation gauge. Here, $[0,1)$ means $\geq 0$ and $<1 \mathrm{~m} \mathrm{~s}^{-1}$.

particularly clear for the Hotplate precipitation gauge compared with the other gauges in Figs. 5 and 6 where the width of the box plot between the 25 th and the 75 th percentile remains mostly constant with increasing wind speed while this range increases and/or varies more with wind speed for the other types of gauges (adjusted or not adjusted).

\section{b. Conclusions}

To conclude, this analysis reveals that the scatter in the collection efficiency for a Hotplate precipitation gauge is significantly smaller than the scatter in collection efficiency from commonly used weighing gauges, even those surrounded by an Alter shield. Specific key results are as follows:

- The collection efficiency of the unadjusted Hotplate precipitation measurements is relatively constant and close to $100 \%$ for wind speeds up to $5 \mathrm{~m} \mathrm{~s}^{-1}$. On the other hand, the collection efficiency of other gauge shield configurations (such as an Alter shielded Geonor or Pluvio gauge) decreases significantly with increasing wind speed. This superior performance of the Hotplate precipitation gauge is likely due to its relatively small impact on snowflake trajectories as compared to catch type precipitation gauges as a result of the Hotplate's aerodynamic shape (Cauteruccio 2020). It is important to note that the unadjusted Hotplate precipitation gauge measurements used the linear correction of Rasmussen et al. (2011). This correction uses the wind measured by the
Hotplate precipitation gauge itself because snow tends to slide off the plate in high wind conditions. In contrast, this study uses wind speed measured by an external sensor to adjust the precipitation for wind undercatch.

- The scatter in the data (RMSE) collected by the Hotplate precipitation gauge is mainly constant with wind speed (up to
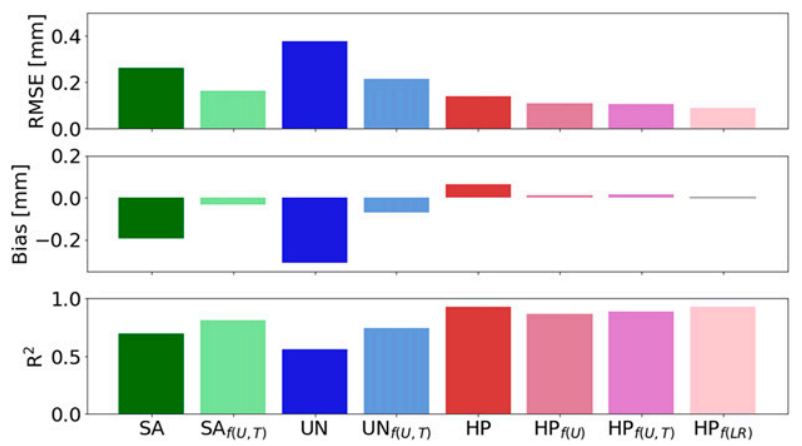

FIG. 9. RMSE, bias, and $R^{2}$ computed with the amount of precipitation unadjusted and adjusted for each instrument $\left(T<-2^{\circ} \mathrm{C}\right)$. UN and $\mathrm{UN}_{f(U, T)}$ denote the unadjusted and adjusted measurements of the unshielded Geonor, respectively. The same notation is used for the Geonor in the single-Alter shield (SA) and Hotplate precipitation gauge (HP). The unadjusted HP measurements (red bars, HP) followed the wind correction of Rasmussen et al. (2011), who used the internal wind measurements of the HP precipitation gauge. 
$5 \mathrm{~m} \mathrm{~s}^{-1}$ ) whereas it generally increases with wind speed for other types of gauges. The RMSE at $0 \mathrm{~m} \mathrm{~s}^{-1}$ depends mainly on the uncertainty of the instruments, the gauge-shield configuration, and the snow accumulation variability at the site.

- The overall scatter in the data (RMSE) is lower for the unadjusted Hotplate precipitation gauge measurements than for the adjusted Geonor measurements in a single Alter and the unshielded Geonor. The RMSE systematically increases with wind speed for the single Alter and unshielded Geonor gauges but not for the Hotplate precipitation gauge.

- The Hotplate precipitation gauge can be adjusted using measured wind speed, 2-m air temperature, as well as using a simple linear regression. The linear regression leads to an improved RMSE and bias.

Snowfall measurements with a Hotplate precipitation gauge have less scatter in the data at a given wind speed compared to standard weighing gauges. Similar datasets were also collected at two other WMO SPICE sites (Sodankyla, Finland, and Haukeliseter, Norway), and we plan to extend this analysis to the lower and higher wind speed conditions present at these sites. The current analysis focuses on wind speeds typically present during midlatitude snow storms. Further studies should be conducted on the impact of the solid precipitation types on the collection efficiency of the Hotplate precipitation gauge. Finally, this study contributes to a better understanding of precipitation gauge collection efficiency for solid precipitation and shows the added value of using new technology that minimally alters the upstream flow field to accurately measure snowfall.

Acknowledgments. The research was funded by the Natural Sciences and Engineering Research Council of Canada (NSERC) Discovery Grant, the Canadian Research Chair Tier 2, and the NCAR Water Cycle System program. The authors thank Scott Landolt, Joshua Lave, and John Kochendorfer for useful discussion and sharing their data.

Data availability statement. Data from the Marshall Test site are available in the supplement file provided with Colli et al. (2020). WMO SPICE and OTT Parsivel ${ }^{2}$ data are available upon request to the Research Application Lab of the National Center for Atmospheric Research (NCAR).

\section{REFERENCES}

Boudala, F. S., G. A. Isaac, R. Rasmussen, S. G. Cober, and B. Scott, 2014: Comparisons of snowfall measurements in complex terrain made during the 2010 Winter Olympics in Vancouver. Pure Appl. Geophys., 171, 113-127, https:// doi.org/10.1007/s00024-012-0610-5.

Cauteruccio, A., 2020: The role of turbulence in particle-fluid interaction as induced by the outer geometry of catchingtype precipitation gauges. Ph.D. thesis, University of Genoa, 176 pp.

Colli, M., L. G. Lanza, R. Rasmussen, and J. M. Thériault, 2016a: The collection efficiency of shielded and unshielded precipitation gauges. Part I: CFD airflow modeling. J. Hydrometeor., 17, 231-243, https://doi.org/10.1175/JHM-D-15-0010.1. $-\ldots, \ldots$, and $\_$- 2016b: The collection efficiency of shielded and unshielded precipitation gauges. Part II: Modeling particle trajectories. J. Hydrometeor., 17, 245255, https://doi.org/10.1175/JHM-D-15-0011.1.

—_, M. Stagnaro, L. G. Lanza, R. Rasmussen, and J. M. Thériault, 2020: Adjustments for wind-induced undercatch in snowfall measurements based on precipitation intensity. J. Hydrometeor., 21, 1039-1050, https://doi.org/10.1175/ JHM-D-19-0222.1.

Goodison, B. E., P. Y. T. Louie, and D. Yang, 1998: World Meteorological Organization Solid Precipitation Measurement Intercomparison. WMO Instruments and Observing Methods Rep. 67, 318 pp., https://www.wmo.int/pages/prog/www/IMOP/ publications/IOM-67-solid-precip/WMOtd872.pdf.

Groisman, P. Y., V. V. Koknaeva, T. A. Belokrylova, and T. R. Karl, 1991: Overcoming biases of precipitation measurement: A history of the USSR experience. Bull. Amer. Meteor., 72, 1725-1733, https://doi.org/10.1175/1520-0477(1991)072<1725: OBOPMA $>2.0$.CO;2.

Kochendorfer, J., and Coauthors, 2017a: The quantification and correction of wind-induced precipitation measurement errors. Hydrol. Earth Syst. Sci., 21, 1973-1989, https://doi.org/10.5194/ hess-21-1973-2017.

_ , and Coauthors, 2017b: Analysis of single-Alter-shielded and unshielded measurements of mixed and solid precipitation from WMO-SPICE. Hydrol. Earth Syst. Sci., 21, 3525-3542, https://doi.org/10.5194/hess-21-3525-2017.

_ functions for weighing precipitation gauges in WMO-SPICE. Hydrol. Earth Syst. Sci., 22, 1437-1452, https://doi.org/10.5194/ hess-22-1437-2018.

Leroux, N. R., J. M. Thériault, and R. Rasmussen, 2021: Improvement of snow gauge collection efficiency through a knowledge of solid precipitation fall speed. J. Hydrometeor., https://doi.org/10.1175/ JHM-D-20-0147.1, in press.

Nitu, R., and Coauthors, 2018: World Meteorological Organization Solid Precipitation Intercomparison Experiment (SPICE) (2012-2015). WMO Instruments and Observing Methods Rep. 131, 1445 pp., https://library.wmo.int/doc_num.php?explnum_id=5686.

Rasmussen, R. M., J. Hallett, R. Purcell, S. D. Landolt, and J. Cole, 2011: The hotplate precipitation gauge. J. Atmos. Oceanic Technol., 28, 148-164, https://doi.org/10.1175/2010JTECHA1375.1.

_- , and Coauthors, 2012: How well are we measuring snow: The NOAA/FAA/NCAR winter precipitation test bed. Bull. Amer. Meteor., 93, 811-829, https://doi.org/10.1175/ BAMS-D-11-00052.1.

Rauch, W., N. Thurner, and P. Harremoes, 1998: Required accuracy of rainfall data for integrated urban drainage modeling. Water Sci. Technol., 37, 81-89, https://doi.org/10.2166/wst.1998.0441.

Sevruk, B., 1982: Methods of correction for systematic error in point precipitation measurement for operational use. Operational Hydrology Rep. 21, WMO Rep. 589, 91 pp.

_ 1996: Adjustment of tipping-bucket precipitation gauge measurements. Atmos. Res., 42, 237-246, https://doi.org/10.1016/01698095(95)00066-6.

Thériault, J. M., R. Rasmussen, K. Ikeda, and S. Landolt, 2012: Dependence of snow gauge collection efficiency on snowflake characteristics. J. Appl. Meteor. Climatol., 51, 745-762, https:// doi.org/10.1175/JAMC-D-11-0116.1.

Wolff, M. A., K. Isaksen, A. Petersen-Øverleir, K. Ødemark, T. Reitan, and R. Brækkan, 2015: Derivation of a new continuous adjustment function for correcting wind-induced loss of solid precipitation: Results of a Norwegian field study. 
Hydrol. Earth Syst. Sci., 19, 951-967, https://doi.org/10.5194/ hess-19-951-2015.

Yang, D., 2014: Double Fence Intercomparison Reference (DFIR) vs. Bush gauge for "true" snowfall measurement. J. Hydrol., 509, 94-100, https://doi.org/10.1016/j.jhydrol.2013.08.052.

, and Coauthors, 1995: Accuracy of Tretyakov precipitation gauge: Result of WMO intercomparison. Hydrol. Processes, 9 , 877-895, https://doi.org/10.1002/hyp.3360090805.
- and Coauthors, 2001: Compatibility evaluation of national precipitation gage measurements. J. Geophys. Res., 106, 14811491, https://doi.org/10.1029/2000JD900612.

Zelasko, N., A. Wettlaufer, B. Borkhuu, M. Burkhart, L. S. Campbell, W. J. Steenburgh, and J. R. Snider, 2018: Hotplate precipitation gauge calibrations and field measurements. Atmos. Meas. Tech., 11, 441-458, https://doi.org/10.5194/amt11-441-2018. 